OPEN ACCESS

Edited by:

Cyril Corbet,

Catholic University of Louvain,

Belgium

Reviewed by:

Paolo E. Porporato,

University of Turin, Italy

Clelia Madeddu,

Università Degli Studi di Cagliari, Italy

*Correspondence:

Gaetano Cairo

gaetano.cairo@unimi.it

Specialty section:

This article was submitted to

Pharmacology of Anti-Cancer Drugs,

a section of the journal

Frontiers in Oncology

Received: 21 December 2018

Accepted: 22 February 2019

Published: 19 March 2019

Citation:

Recalcati S, Correnti M, Gammella E,

Raggi $C$, Invernizzi $P$ and Cairo $G$

(2019) Iron Metabolism in Liver Cancer

Stem Cells. Front. Oncol. 9:149.

doi: 10.3389/fonc.2019.00149

\section{Iron Metabolism in Liver Cancer Stem Cells}

\author{
Stefania Recalcati ${ }^{1}$, Margherita Correnti ${ }^{2}$, Elena Gammella ${ }^{1}$, Chiara Raggi ${ }^{2,3}$, \\ Pietro Invernizzi ${ }^{4}$ and Gaetano Cairo ${ }^{1 *}$
}

${ }^{1}$ Department of Biomedical Sciences for Health, University of Milan, Milan, Italy, ${ }^{2}$ Humanitas Clinical and Research Center, IRCCS, Rozzano, Italy, ${ }^{3}$ Department of Experimental and Clinical Medicine, University of Florence, Florence, Italy, ${ }^{4}$ Division of Gastroenterology, Department of Medicine and Surgery, Center for Autoimmune Liver Diseases, San Gerardo Hospital, University of Milano-Bicocca, Monza, Italy

Cancer stem cells (CSC) which have been identified in several tumors, including liver cancer, represent a particular subpopulation of tumor cells characterized by properties similar to those of adult stem cells. Importantly, CSC are resistant to standard therapies, thereby leading to metastatic dissemination and tumor relapse. Given the increasing evidence that iron homeostasis is deregulated in cancer, here we describe the iron homeostasis alterations in cancer cells, particularly in liver CSC. We also discuss two paradoxically opposite iron manipulation-strategies for tumor therapy based either on iron chelation or iron overload-mediated oxidant production leading to ferroptosis. A better understanding of iron metabolism modifications occurring in hepatic tumors and particularly in liver CSC cells may offer new therapeutic options for this cancer, which is characterized by increasing incidence and unfavorable prognosis.

Keywords: iron, liver cancer, cancer stem cells, ferroptosis, chelators

\section{INTRODUCTION}

The phenotypical and functional heterogeneity of cells within tumors can be explained by both the conventional mechanism centered on clonal evolution and a model based on the presence of cancer stem cells (CSC) which over the last decade received support by increasing experimental evidence. CSC have similar properties to adult stem cells, such as the ability for unlimited self-renewal and differentiation and are believed to be a major source of cancer initiation and progression, thus resulting in a heterogeneous tumor cell progeny (1-3). Moreover, CSC are characterized by drug resistance, an element allowing tumors to survive therapies. In fact, the relatively quiescent CSC can escape cell death after standard chemotherapy treatments, which preferentially eliminate rapidly proliferating cells; as a consequence, the remaining CSC may lead to cancer relapse and metastasis, whose treatment is more complex and often unsatisfactory. Notably, it is now also realized that some of the alterations of iron homeostasis that have recently emerged as key factors in cancer growth and progression are present also in CSC [reviewed in (4)]. In this Review, we discuss current knowledge of the role of iron as a key factor in cancer development, particularly in liver and hepatic CSC, and we also address iron-centered therapeutic approaches.

\section{PRIMARY LIVER CANCER AND CANCER STEM CELLS}

Primary liver cancer (PLC) is the fifth most common cancer worldwide and the second most frequent leading cause of cancer-related mortality and its incidence and mortality are increasingat a fast rate, especially in western countries $(5,6)$. Hepatocellular carcinoma (HCC) and 
cholangiocarcinoma (CCA) represent the two major forms of PLC, and account for $~ 90$ and 5\% of all primary liver tumors, respectively $(5,6)$.

HCC arises from malignant transformation of hepatocytes and is often associated with known risk factors, such as excessive alcohol intake, infection with hepatitis $\mathrm{B}$ virus (HBV) or hepatitis $\mathrm{C}$ virus (HCV), aflatoxin B1 intake, fatty infiltration, autoimmune liver diseases and alterations of iron metabolism leading to hepatic iron accumulation like hemochromatosis (5, 7). Unlike the HCV-related HCCs, the incidence of HCC linked to the metabolic syndrome is increasing, principally due to obesity epidemic continuous rising (8).

On the other hand, CCA arise from neoplastic transformation of intra- and extra-hepatic biliary epithelial cells (cholangiocytes) and the frequency of its established risk factors mostly differs depending on geographic area (9). For example, infection with liver flukes (Opisthorchis viverrini and Clonorchis sinensis) is a common risk factor for CCA development in Southeast Asia $(6,10)$. Conversely, primary sclerosing cholangitis (PSC) is the most common predisposing condition for CCA in the western countries (6). HBV or HCV infection and cirrhosis have been also proposed as potential etiologies of CCA, shared with HCC $(6,7)$.

While liver transplantation, surgical resection and locoregional therapies are possible curative options at early phases, the majority of PLC patients unfortunately present advanced stages of the disease, for which treatments are very limited and the prognosis remains unfavorable $(5,11)$.

Like most other solid tumors, PLC are characterized by an extensive clinical and pathobiological heterogeneity in terms of cellular morphologies as well as genetic and epigenetic landscape (12-14). Such intra-tumor heterogeneity may reflect the presence of different clonal subpopulations exhibiting differential sensitivity to drugs $(7,12,13)$. In this respect, the recent advent of the CSC hypothesis has added a new level of complexity in understanding PLC heterogeneity and drug resistance. According to this model, CSC are thought to drive tumor growth and progression, as well as tumor metastasis, recurrence and therapy resistance, representing the unique unit of selection within the tumor (2, 15-17). Interestingly, a new "CSC plasticity model" has been proposed, further increasing the complexity of tumor biology. According to this theory, the different tumor cell subpopulations are highly plastic and dynamic, continuously switching between non-CSC and CSC phenotypes, depending on various intrinsic and extrinsic stimuli (18).

While the CSC existence has been confirmed in HCC (19-22) and recently also in CCA $(23,24)$, no consensus has yet been reached regarding the origin of hepatic CSC. In addition to the classical idea that CSC originate from normal liver resident stem cells (1), it is now become accepted that CSC may originate also from more committed progenitor cells and mature differentiated tumor cells through a reprogramming process $(11,25,26)$. These considerations go hand in hand with the unsettled debate about the true nature of the PLC cell-of-origin, about which a consensus has not been reached, yet.

Another open question concerns the identification of a common recognized method for isolation and subsequent characterization of liver CSC. During the last years several attempts have been made to obtain a cell population enriched in liver CSC using a variety of techniques. The antigenic approach, which is one of the first methods used to isolate CSC, relies on surface CSC markers detection, including CD133, CD44, OV6, CD90, EpCAM, CD13, CD24, CD47 (27). However, the antigenic approach has several shortcomings, such as lack of clearly defined surface markers specific for individual tumor type, such as PLC, and the fact that different cancer cell populations with tumor-initiating activity can be isolated using different markers within a tumor type $(18,28)$. Therefore, none of the proposed markers is specific for liver CSC and universally expressed in all liver CSC (29). In addition, the surface marker expression can diverge depending on the specific context (28). In addition to the classical antigenic approach, there are several assays based on CSC functional properties, including Side Population (SP) analysis, Aldefluor assay and tumor-sphere formation assay (14, 24, 27). All these functional techniques are based on different key CSC features: the first one on the typical drug resistance of CSC $(30,31)$, the second one on the measurement of aldehyde dehydrogenase activity (32) and the last one on long-term selfrenewal capability of CSC $(21,24,33)$. Possibly, a combinatorial strategy might be a valid alternative to isolate a betterdefined PLC stem-like subset, but the gold-standard approach to evaluate CSC tumorigenic potential remains the in vivo approach based on xenotransplantation in immune-deficient mice $(14,24,27)$.

\section{IRON AND LIVER CANCER}

Iron is an essential component of living organisms, as it is necessary for cellular metabolism, replication and growth. However, excess iron can facilitate the generation of the most reactive and toxic forms of oxidants through the Fenton reaction (34); therefore, iron levels are carefully kept within an optimal range at both systemic and cellular levels (Figure 1). The major players in maintaining cellular iron homeostasis are the transferrin receptor (TfR1) that internalizes transferrin-bound iron, ferroportin (Fpn), the only cellular iron exporter, and ferritin that stores excess iron (35) (Figure 2). A number of epidemiological studies indicate a positive association between cancer and high body iron content in the general population (36). Since the liver is the organ where excess iron accumulates (37) and plays an important role in maintaining iron homeostasis, a large body of evidence from human, animal, and in vitro studies supports the positive relation between increased body iron stores and the risk of liver cancer. In fact, HCC is the prevalent tumor found in hemochromatosis patients (38).

Studies investigating cancer risk in subjects undergoing blood transfusion or phlebotomy suggest that iron excess is not merely associated with cancer but plays an active role in carcinogenesis. The biological basis of the association between iron and cancer is double-face as it probably rests in both oxidative stress-mediated DNA damage and availability of the metal to support fast growth (39). Iron may therefore play a role both as an initiator in an early phase and, once malignant change has occurred, as a promoter 


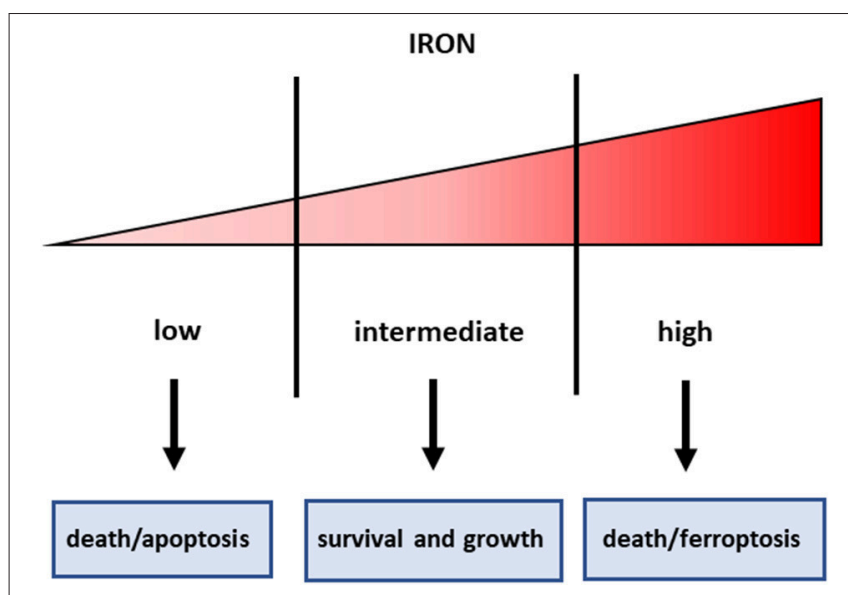

FIGURE 1 | Iron threshold concept. Certain iron levels are required for cell survival and homeostasis, but iron concentrations too low lead to apoptotic cell death, whereas excess iron equally triggers cell death.

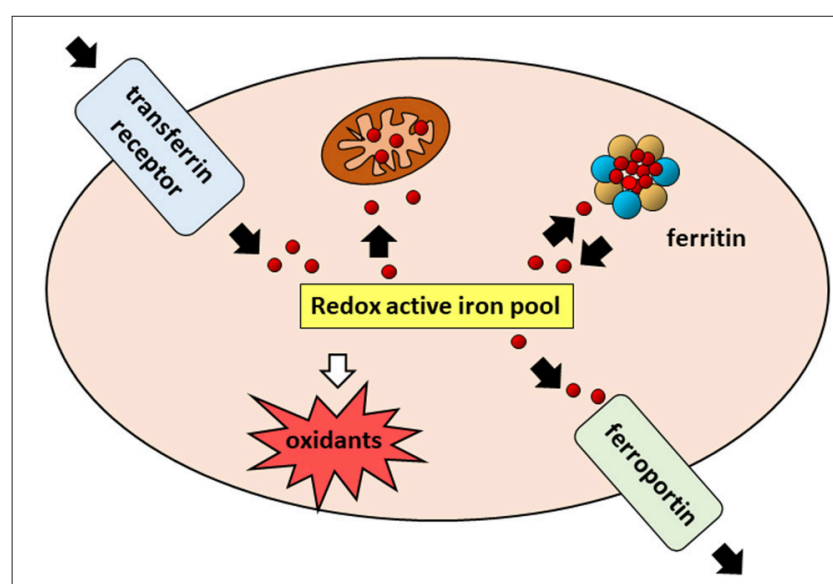

FIGURE 2 | Cellular iron pathways in a nutshell. Transferrin bound iron, internalized through endocytosis of the transferrin receptor (TfR1), enters a pool of redox-active iron whose concentration is kept under control by mechanisms ensuring that the iron which is not used for biochemical processes, particularly in mitochondria, is either safely stored in cytoplasmic ferritin or exported by ferroportin.

that allows the transformed cell to fully express its potential of unrestricted growth.

In addition, recent studies showed that both systemic and cellular iron metabolism is altered in tumors (40). In general, given the high iron needs of tumor cells to sustain cell proliferation, the alterations of iron trafficking in cancer cells lead to iron acquisition. To this purpose, cancer cells usually increase iron uptake, for example by up-regulating TfR1, decrease iron release by inhibiting Fpn, or both. Several studies have demonstrated that these alterations of cellular iron metabolism are directly dependent on the action of oncogenes and tumor suppressors (39). Notably, the "iron addiction" of tumors was confirmed by the analysis of different cell lines using a novel method (41), which showed that cancer cells had significantly increased redox-active iron pools compared to non-tumorigenic cells. The role of iron in cancer is not related only to the "iron seeking" phenotype of most cancer cells. In fact, iron levels can modulate apoptosis in multiple ways, for example by affecting the alternative splicing of Fas/CD95 transcripts between the pro-apoptotic and anti-apoptotic isoforms (42). Moreover, the p53 pathway that regulates cell cycle and apoptosis interacts with iron metabolism in a complicated crosstalk that remains to be completely explained (43). As an example of opposing observations regarding the involvement of iron and p53 in the pathogenesis of HCC, it has been shown that exposure to iron down-regulated MDM2, the ubiquitin ligase which leads to degradation of p53 (44), whereas another study found decreased p53 protein levels in the liver of iron overloaded mice (45).

Iron metabolism has been investigated in rodent models of hepatic carcinogenesis as well as in regenerating liver, which represents an excellent example of controlled liver proliferation and hence a powerful model system to get insights into the processes leading to hepatocarcinogenesis. Similarly to other types of growing cells, increased expression of TfR1 has been found in rat liver preneoplastic nodules and $\operatorname{HCC}(46,47)$, as well as in regenerating liver cells (48), probably in order to insure sufficient iron to sustain cell proliferation. Recently, a study investigating iron metabolism gene expression and prognostic features of HCC found that TfR1 are more expressed in HCC than in surrounding liver and correlate with poorer prognosis (49). Researches also addressed the role of iron in CCA, a severe liver tumor with limited therapeutic possibilities, concluding that high expression of TfR1, with consequent iron uptake, contributes to CCA progression and poorer clinical outcomes (50). Accordingly, we showed that elevated iron content is a negative prognostic factor in CCA patients (51).

However, recent studies indicated that altered expression of proteins of iron metabolism like TfR1 in tumor cells is not only a system to acquire more iron but may impinge on tumor growth in an iron-independent manner. In fact, in line with the demonstrated interaction of TfR1 with ligands other than transferrin (Tf) (52), it appears that, in addition to its role in iron uptake, TfR1 activates signaling pathways and has a role in apoptosis, a key process in cancer development. For example, TfR1, by activating JNK upon phosphorylation by Src, impairs apoptosis and thereby increases breast cancer cell survival (53). In addition, the interaction of TfR1 with Tf may also have roles which are different from iron uptake but are still important for tumor cell growth; in fact TfR1 appears to be implicated in epithelial mesenchymal transition (EMT) (54), which is an important process for cancer progression, and metastatic growth (55).

Over the last years, a number of studies have shown that the levels of Fpn are reduced in several cancer cells compared to their nonmalignant counterparts, so that Fpn downregulation appears as a common strategy that a variety of cancer cells adopt to enhance intracellular iron availability (39). Interestingly, Fpn expression is a strong and independent predictor of prognosis in different tumor types (39). Dysregulation of the hepcidin/Fpn axis may also play a relevant role in liver tumors. Recently, it has been shown that hypermethylation of specific sequences 
in the promoter region of the gene coding for hepcidin, the liver hormone that regulates iron homeostasis by inhibiting Fpnmediated iron export, results in transcriptional downregulation of hepcidin expression in HCC (56). A similar effect was found in a model of rat liver carcinogenesis, in which the downregulation of hepcidin and the consequent increase of Fpn-mediated iron release may underlie the decreased intracellular levels of iron in preneoplastic foci (57). This could be a specific feature of hepatic cancer, as Fpn is usually repressed in cancer cells and low iron levels are not attributed to increased iron export but to higher consumption. However, we found significantly reduced Fpn mRNA levels in tumor samples from CCA patients compared to matched surrounding liver, suggesting that also in PLC elevated iron content is a negative prognostic factor (51).

The reprogramming of iron metabolism in tumors comprises the repression of the iron storage protein ferritin, as a mean to increase iron availability for the high requirements of cancer cell (58). Conversely, a tumor-suppressive role for ferritin has been shown in several types of cancer, such as breast and colorectal cancer. Ferritin expression appears to be directly modulated by both oncogenes, which down-regulate ferritin, and tumor suppressors, which induce ferritin expression [reviewed in Torti (39)]. Accordingly, a recent study highlighted a new mechanism based on a complex miRNA network by which oncogenic miRNAs inhibit the expression of $\mathrm{H}$ ferritin and its pseudogenes, thus leading to prostate cancer growth (59). Ferritin expression in liver cancer has been investigated by a number of studies in rodent models of hepatocarcinogenesis and in human hepatomas (60), but a coherent picture has not emerged, probably as the result of different experimental approaches and models, but also because of the multiple mechanisms of regulation of this protein, which is affected by iron status, differentiation, growth rate, inflammation, etc. (61). Therefore, whether increased ferritin levels in HCC patients merely reflect increased accumulation of iron, which is the actual carcinogen, or play an active role in malignant transformation is still unknown.

\section{LIVER CSC AND IRON}

Over the last years, several investigations found increased iron content in CSC of several types of tumors and also showed that altered iron trafficking is functional to the role of CSC in tumor growth. Indeed, variations of iron levels in CSC were achieved by specific modulation of the expression of genes of iron metabolism; in particular, TfR1-dependent iron uptake is induced whereas Fpn-mediated iron export is down-modulated. In most studies, the enhanced iron content was mirrored by high levels of the iron storage protein ferritin $(4,62)$.

Support to the idea that higher iron levels have a functional role in CSC formation and the maintenance of stemness was provided by evidence that iron chelation inhibited tumor spheres (a proxy of CSC) formation in several types of cancers (4). Additionally, manipulation of iron levels modulated the expression of typical stemness markers (4). Of course, it is also conceivable that alterations of iron homeostasis induced by genes related to CSC, such as Myc-mediated inhibition of ferritin expression (63), cooperate in order to disrupt iron homeostasis in CSC.

Notably, in vivo studies showing higher tumorigenic capacity of iron-rich tumor spheres in mouse xenograft tumor models confirmed the role of iron (64-66). Moreover, poorer prognosis for human tumors with altered expression of iron proteins in CSC is a common finding $(51,64,66-68)$.

As it regards the role of iron in liver CSC, knowledge is limited. We recently showed that the regulation of iron homeostasis is profoundly different if CCA cell lines are cultured under conditions inducing the formation of tumor spheres, as compared to CCA cells growing in monolayers. In particular, we found high $\mathrm{H}$ ferritin levels and TfR1 expression accompanied by diminished Fpn transcription, a pattern leading to elevated iron content. Moreover, this finding was mirrored by data showing a trend toward shorter survival in CCA patients with high expression of ferritin and hepcidin (51).

\section{IRON AND CANCER THERAPY}

In consideration of the role of iron in cancer, and particularly in CSC that are resistant to radio and chemotherapy, manipulation of iron levels appears a promising therapeutic strategy. Given the double-edged sword property of iron in controlling cell fate (Figure 1), both iron chelation (in order to starve tumor cells for this essential micronutrient) and iron overload (in order to exploit iron toxicity) have been proposed for cancer treatment, but there are still several concerns for the use of both strategies. In fact, the threshold above which iron levels are no longer supportive of growth but become toxic is not well-defined, even though recent findings indicated that the functional iron concentration that allows in vitro cell proliferation is very low (i.e., in the nanomolar range) (69). Iron chelation has been used in several types of cancer, including HCC (70). However, since desferrioxamine, an iron chelator in use since the nineties, has a rather poor bioavailability, limited success in treating cancer has been obtained with iron chelators so far, though more recently developed oral iron chelators like deferasirox showed some effect in leukemia patients (71). The major mechanisms by which sequestration of intracellular iron by classical iron chelators targets tumor cells are:(i) inhibition of the iron-containing ribonucleotide reductase, the rate-limiting enzyme for DNA synthesis, (ii) cyclin dependent kinases-mediated induction of cell cycle arrest, iii) activation of metastasis and tumor suppressor genes, such as NDRG1 and p53, respectively. Moreover, recent data indicate that chelators can also suppress cancer by inhibiting the EMT, a key characteristic of CSC (72). Since the prolyl hydroxylases controlling the levels of the hypoxia inducible factors (HIF) are iron dependent enzymes, iron chelators induce HIF and its numerous target genes (73). We have shown that HIF-1 is involved in the protective effect exerted by the iron chelator dexrazoxane against anthracycline cardiotoxicity (74). Therefore, one may legitimately wonder whether iron chelators may have a similar effect in cancer cells, thus limiting the therapeutic effect of anticancer drugs. 
A new class of iron chelators, such as the thiosemicarbazone Dp44m, which were reported to inhibit the proliferation of cancer cell lines in vitro by inducing the expression of $\mathrm{p} 21$, a cyclin-dependent kinase inhibitor involved in cell cycle arrest (75), appear promising. Opposite to conventional iron chelators like desferrioxamine, Dp44m is a tridentate ligand that forms redox active iron complexes leading to increased oxidant levels and cytotoxicity (76). These compounds also limit the growth of tumor xenografts in nude mice and have entered clinical trials, but their effect on CSC has not been tested. However, considering the well-known drug resistance of CSC, it is worth to mention that lysosomal-targeted Dp44m prevents the sequestration of a chemotherapeutic anthracycline like doxorubicin in lysosomes, which is triggered by the stressful environment of the tumor (77). Through this mechanism, which allows doxorubicin to exert its toxic effects in the nucleus and the mitochondria, this chelator may thus favor the action of anticancer drugs. In the same line, a recent study showed that a novel iron chelator, DpdtC (di-2pyridylketone hydrazone dithiocarbamate) can induce lysosomal oxidant production and growth inhibition of HCC cell lines (78).

The use of these compounds represents therefore an approach similar to that relying on the toxic side of iron for killing cancer cells (see below). This strategy gained momentum after the discovery of ferroptosis, a form on non-apoptotic cell death caused by iron-dependent production and accumulation of reactive and toxic hydroperoxides (79). Iron plays a dual role in ferroptosis, as iron on the one hand can promote Fenton chemistry and on the other hand stimulate the activity of the iron-dependent enzyme lipoxygenases that contribute to ferroptosis by degrading polyunsaturated fatty-acid-containing phospholipids (80). Malignant cells generate high levels of oxidants as by-products of the biosynthesis of macromolecules and must balance iron uptake for proliferation with the risk of generating oxidative stress (81). Since most ferroptosis-inducing agents have limited use in vivo due to low bioavailability (82), iron may thus have potential to trigger ferroptotic cell death in cancer, as high iron concentrations may push malignant cells beyond the breaking point of oxidative stress tolerance (34). Interestingly, it has been shown that iron is required to induce ferroptosis also in drug-resistant "persister" cancer cells, thus showing therapeutic promise to eliminate this pool of tumor cells characterized by non-mutational drug-tolerance (83).

Alternatively, iron may potentiate the effect of anticancer drugs like the multikinase inhibitor sorafenib, which is used for treating HCC. In fact, the evidence that iron chelation protected HCC cells against iron-dependent oxidative stress caused by sorafenib indicates that ferroptosis can represent an inhibitory mechanism of the growth of liver cancer cells (84). A further indication of the role of iron and ferroptosis in PLC was provided by a study showing that ferritin induction protects HCC cells from ferroptosis (85).

Triggering ferroptosis appears a promising approach also to attack CSC, which represent a negative factor for cancer outcome (86). Ovarian CSC showed higher sensitivity to ferroptosis than non-tumorigenic ovarian stem cells (66) and a recent study showed that in breast CSC exposure to salinomycin and its derivatives leads to lysosomal iron accumulation, oxidants production and ferroptosis (87). Similarly, increased intracellular iron levels provided by ferritin degradation can lead glioblastoma cells to ferroptosis (64). However, a number of recent studies have shown that CSC are iron-rich and iron-dependent (4); therefore, enhancing cellular iron may not always be an effective strategy to eliminate CSC selectively. Moreover, in general, low levels of oxidants have been reported in CSC, making difficult the approach based on targeting iron-dependent, oxidant related pathways in CSC. In fact, we found that sphere-forming CCA cells, in spite of higher levels of oxidants and iron, were more resistant to the ferroptosis inducer erastin than their counterpart growing as monolayer (51). It seems therefore that, before recommending the manipulation of iron homeostasis as a therapeutic tool for targeting tumors and the use of iron supplementation to promote ferroptosis of cancer cells, additional studies are needed to understand the role of iron in the pathways controlling cell death, as iron can possibly promote CSC cell growth, thereby affecting the survival of cancer patients.

It should be also kept in mind that HCC almost always develop in the context of chronic liver disease characterized by persistent damage and inflammation, which can be further stimulated by iron supplementation.

\section{TARGETING IRON TO CANCER CELLS}

Administration of massive quantities of iron successfully killed multiple myeloma cells (88), though these cells, which secrete large amounts of disulfide-rich immunoglobulins and are thus a source of oxidants (89), may be particularly sensitive to irondependent oxidative stress. Iron may also mediate the effect of high i.v. doses of vitamin $\mathrm{C}$ that were reported to kill liver CSC specifically both in vitro and in vivo by promoting oxidant production (90). This paradoxical effect of a recognized antioxidant may be explained by the strong reducing properties of ascorbic acid which, at pharmacological concentrations $(>1 \mathrm{mM})$, maintains iron in the highly reactive ferrous form, thereby increasing, instead of preventing, oxidative stress and cell death.

How to load cancer cells, in particular CSC, with iron in vivo, possibly in a specific way? Oral iron is poorly adsorbed and its uptake is subjected to a strict feed-back regulation (91), therefore, even recurring to novel nanoparticulate ferritin core mimetics (92), it does not appear a promising approach. Parenteral iron preparations, such as dextran iron, have higher efficacy but relatively poor safety due to hypersensitivity reactions. However, new formulations, such as iron gluconate and iron sucrose, do not present toxicity issues and two iv iron compounds prepared with new pharmaceutical technologies are currently approved for the treatment of iron deficiency anemia (93) and could be used in cancer patients. These iron complexes are endocytosed and processed by macrophages within the reticuloendothelial system, mainly in the liver, spleen and bone marrow, but the precise mechanism of recognition and internalization is not fully defined (94). Inside the macrophages, iron is released from the iron-carbohydrate complex in acidic endo-lysosomes through a mechanism incompletely understood 
and subsequently transported to the cytoplasm, where it can be stored in ferritin or exported into the bloodstream by Fpn.

An approach alternative to exogenous iron administration is to impair safe intracellular iron storage, for example by triggering lysosomal ferritin degradation. Indeed, treatment of breast CSC with salinomycin resulted in increased ferritin degradation in lysosomes; the iron released then facilitated oxidants production and ferroptosis (87). An analogous release of catalytic ferrous iron from ferritin led mesothelioma cells to death after exposure to non-thermal plasma, which produces hydroxyl radicals (95). Similarly, artesunate, by enhancing lysosomal ferritin degradation, was able to induce cell death in HCC cell lines (96). Notably, regulated autophagic degradation of ferritin (ferritinophagy) contributes to ferroptosis and was found to occur in primary human hepatic stellate cells obtained from liver tissue of advanced fibrotic patients with HCC, thereby alleviating liver fibrosis (97). However, ferritin is not only, or not always, a source of iron for Fenton chemistry. Iron storage inside ferritin is a protective stratagem against iron-mediated oxidative injury $(34,61)$ and also mitochondrial ferritin shields this important organelle from oxidants damage (98). The relevance of this function in cancer has been shown by a study reporting that high ferritin expression in myeloma cells is directly related to increased resistance to oxidants generated by exposure to the proteasome inhibitor bortezomib (88). A similar effect was found in HCC cells in which oxidative stress mediated induction of ferritin protected from ferroptosis (85).

The alterations of iron homeostasis seem to involve not only cancer cells, but also other cell types of the tumor microenvironment, particularly macrophages. In response to signals in the tumor microenvironment, tumor-associated macrophages (TAM), which favor tumor growth and progression, often become similar to M2 polarized macrophages endowed with anti-inflammatory activity, which display a gene expression profile characterized by active iron uptake and release and ferritin repression $(99,100)$. Therefore, this kind of iron metabolism in TAM macrophages might promote tumor growth by providing iron to adjacent tumor cells (101). Notably, also CCA CSC prime TAM toward a tumor-promoting phenotype, although iron metabolism has not been explored in this setting (24). However, it should be noted that the TAM population may be heterogeneous (102), as it has been found that in one type of murine prostate cancer, but not in another model of prostate cancer, some TAM contain iron aggregates typical of iron storing macrophages $(103,104)$ and in ovarian cancer TAM presented a prevalence of M1 phenotype (105). In this case, TAM, by sequestering iron, may limit its availability to cancer cells, thus impairing tumor growth. On the other hand, accumulation of an excess of exogenously administered iron, in the same way as an excess of heme iron in hemorrhagic tumor regions (106), may induce a switch of TAM toward the M1 antitumor phenotype, which is associated with the most favorable prognosis, as recently confirmed by extensive immunogenomic analysis of thousands of diverse tumor types (102).

It should be noted that in malignancies induced (or accompanied) by constant damage and chronic inflammation like HCC, two factors can further impinge on iron trafficking: on the one hand, TAM are more M1-like (107) and could restrict iron availability in the microenvironment and exert toxicity against malignant cells; on the other hand, the high hepcidin levels caused by inflammation may weaken Fpn-mediated iron release from macrophages, thus contrasting the iron-donating activity of TAM. These considerations are at odd with the correlation between high hepcidin levels and tumor progression in breast cancer patients $(108,109)$ and poorer prognosis in CCA (51). However, hepcidin may interact with Fpn expressed by both TAM and cancer cells; moreover, other iron transporters like lipocalin2 (110) may be involved.

While the role of iron in TAM, which seems clearly contextdependent, remains to be fully clarified, iron handling by TAM may have therapeutic implications. In fact, a seemingly promising approach relies on the use of iron oxide nanoparticles, a type of nanocarriers used for cancer targeted drug delivery, which are internalized by macrophages, including TAM. In line with in vitro data showing that superparamagnetic iron oxides induce a macrophage shift from the M2 to the M1 subtype (111), a recent study showed that iron oxide nanoparticles inhibited tumor growth indirectly by inducing M1 polarization. Highly increased iron levels in TAM resulted in oxidants production and cancer cell apoptosis (112). While the specific targeting of some species of drug delivering nanoparticles to tumors relies on the higher permeability of leaky blood vessels inside the cancerous mass, thanks to the magnetic properties of iron oxide nanoparticles, a localized external magnetic field can be used to guide these nanoparticles to tumors, thus achieving an improved therapeutic response and reducing side effects.

\section{CONCLUSIONS}

There is growing evidence that iron homeostasis is dysregulated in cancer, including PLC, and over the past few years also insights into the key role of iron in CSC have emerged. CSC show alterations of iron metabolism leading to a phenotype characterized by elevated cellular iron content, so that the expression of their typical features, such as stemness, is inhibited by iron chelators, thus suggesting the use of these compounds for CSC-targeted therapy. On the other hand, most recent therapeutic approaches seem aimed at exploiting the capacity of excess iron to induce ferroptotic cell death in cancer cells. However, given the involvement of iron in many important pathophysiological settings, it should be considered that we need to better understand how manipulation of iron levels to contrast tumor growth may interfere with iron homeostasis in healthy tissues or worsen conditions accompanying cancer, such as inflammation or anemia. Moreover, unfortunately, the mechanism(s) underlying the redox regulation in CSC are still not fully understood, as indicated by the higher resistance of CCA CSC to ferroptosis despite a higher basal oxidative stress condition (51).

Despite the still limited understanding of many processes, the increasing recognition of the importance of iron in cancer biology offers new chances to unravel tumor pathogenesis and thus develop more effective iron-centered therapeutic strategies against liver cancer. 


\section{AUTHOR CONTRIBUTIONS}

MC, SR, and GC wrote the manuscript.

CR,

$\mathrm{EG}$, and PI made significant revisions to the manuscript. All authors read and approved the final manuscript.

\section{REFERENCES}

1. Beachy PA, Karhadkar SS, Berman DM. Tissue repair and stem cell renewal in carcinogenesis. Nature. (2004) 432:324-31. doi: 10.1038/nature03100

2. Plaks V, Kong N, Werb Z. The cancer stem cell niche: how essential is the niche in regulating stemness of tumor cells? Cell Stem Cell. (2015) 16:225-38. doi: 10.1016/j.stem.2015.02.015

3. Magee JA, Piskounova E, Morrison SJ. Cancer stem cells: impact, heterogeneity, and uncertainty. Cancer Cell. (2012) 21:283-96. doi: 10.1016/j.ccr.2012.03.003

4. Recalcati S, Gammella E, Cairo G. Dysregulation of iron metabolism in cancer stem cells Free Radic Biol Med. (2018) 133:216-220. doi: 10.1016/j.freeradbiomed.2018.07.015

5. El-Serag HB. Hepatocellular carcinoma. N Engl J Med. (2011) 365:1118-27. doi: 10.1056/NEJMra1001683

6. Banales JM, Cardinale V, Carpino G, Marzioni M, Andersen JB, Invernizzi $\mathrm{P}$, et al. Expert consensus document: cholangiocarcinoma: current knowledge and future perspectives consensus statement from the european network for the study of cholangiocarcinoma (ENS-CCA). Nat Rev Gastroenterol Hepatol. (2016) 13:261-80. doi: 10.1038/nrgastro. 2016.51

7. Castelli G, Pelosi E, Testa U. Liver cancer: molecular characterization, clonal evolution and cancer stem cells. Cancers. (2017) 9:E127. doi: $10.3390 /$ cancers 9090127

8. Sullivan KM, Kenerson HL, Pillarisetty VG, Riehle KJ, Yeung RS. Precision oncology in liver cancer. Ann Transl Med. (2018) 6:285. doi: 10.21037/atm.2018.06.14

9. Blechacz B, Gores GJ. Cholangiocarcinoma: advances in pathogenesis, diagnosis, and treatment. Hepatology. (2008) 48:308-21. doi: $10.1002 /$ hep. 22310

10. Rizvi S, Gores GJ. Pathogenesis, diagnosis, and management of cholangiocarcinoma. Gastroenterology. (2013) 145:1215-29. doi: 10.1053/j.gastro.2013.10.013

11. Oikawa T. Cancer Stem cells and their cellular origins in primary liver and biliary tract cancers. Hepatology. (2016) 64:645-51. doi: 10.1002/hep.28485

12. Heppner GH, Miller BE. Tumor heterogeneity: biological implications and therapeutic consequences. Cancer Metasta Rev. (1983) 2:5-23. doi: 10.1007/BF00046903

13. Kreso A, Dick JE. Evolution of the cancer stem cell model. Cell Stem Cell. (2014) 14:275-91. doi: 10.1016/j.stem.2014.02.006

14. Raggi C, Mousa HS, Correnti M, Sica A, Invernizzi P. Cancer stem cells and tumor-associated macrophages: a roadmap for multitargeting strategies. Oncogene. (2015) 35:671-82. doi: 10.1038/onc.2015.132

15. O'Brien CA, Pollett A, Gallinger S, Dick JE. A human colon cancer cell capable of initiating tumour growth in immunodeficient mice. Nature. (2007) 445:106-10. doi: 10.1038/nature05372

16. Borovski T, De Sousa E Melo F, Vermeulen L, Medema JP. Cancer stem cell niche: the place to be. Cancer Res. (2011) 71:634-9. doi: 10.1158/0008-5472.CAN-10-3220

17. Meacham CE, Morrison SJ. Tumour heterogeneity and cancer cell plasticity. Nature. (2013) 501:328-37. doi: 10.1038/nature12624

18. da Silva-Diz V, Lorenzo-Sanz L, Bernat-Peguera A, Lopez-Cerda M, Muñoz P. Cancer cell plasticity: impact on tumor progression and therapy response. Semin Cancer Biol. (2018) 53:48-58. doi: 10.1016/j.semcancer.2018.08.009

19. Yamashita T, Ji J, Budhu A, Forgues M, Yang W, Wang HY, et al. EpCAM-positive hepatocellular carcinoma cells are tumor-initiating cells with stem/progenitor cell features. Gastroenterology. (2009) 136:1012-24. doi: 10.1053/j.gastro.2008.12.004

\section{FUNDING}

Work in the author's laboratories was supported by grants from the Ministry of University and Research (MIUR), COFIN project, to GC; Italian Foundation of Cancer Research (AIRC) award (MFGA17588) to CR; MC was supported by AIRC.

20. Marquardt JU, Raggi C, Andersen JB, Seo D, Avital I, Geller D, et al. Human hepatic cancer stem cells are characterized by common stemness traits and diverse oncogenic pathways. Hepatology. (2011) 54:1031-42. doi: 10.1002/hep. 24454

21. Raggi C, Factor VM, Seo D, Holczbauer A, Gillen MC, Marquardt JU, et al. Epigenetic reprogramming modulates malignant properties of human liver cancer. Hepatology. (2014) 59:2251-62. doi: 10.1002/hep.27026

22. Ma S, Chan KW, Hu L, Lee TK, Wo JY, Ng IO, et al. Identification and characterization of tumorigenic liver cancer stem/progenitor cells. Gastroenterology. (2007) 132:2542-56. doi: 10.1053/j.gastro.2007.04.025

23. Kokuryo T, Yokoyama Y, Nagino M. Recent advances in cancer stem cell research for cholangiocarcinoma. J Hepato-Biliary-Pancreatic Sci. (2012) 19:606-13. doi: 10.1007/s00534-012-0542-6

24. Raggi C, Correnti M, Sica A, Andersen JB, Cardinale V, Alvaro D, et al. Cholangiocarcinoma stem-like subset shapes tumor-initiating niche by educating associated macrophages. J Hepatol. (2017) 66:102-15. doi: 10.1016/j.jhep.2016.08.012

25. Rais Y, Zviran A, Geula S, Gafni O, Chomsky E, Viukov S, et al. Deterministic direct reprogramming of somatic cells to pluripotency. Nature. (2013) 502:65-70. doi: 10.1038/nature12587

26. Chaffer CL, Brueckmann I, Scheel C, Kaestli AJ, Wiggins PA, Rodrigues LO, et al. Normal and neoplastic nonstem cells can spontaneously convert to a stem-like state. Proc Natl Acad Sci USA. (2011) 108:7950-5. doi: 10.1073/pnas. 1102454108

27. Correnti M, Raggi C. Stem-like plasticity and heterogeneity of circulating tumor cells: current status and prospect challenges in liver cancer. Oncotarget. (2017) 8:7094-115. doi: 10.18632/oncotarget.12569

28. Cardinale V, Renzi A, Carpino G, Torrice A, Bragazzi MC, Giuliante F, et al. Profiles of cancer stem cell subpopulations in cholangiocarcinomas. Am J Pathol. (2015) 185:1724-39. doi: 10.1016/j.ajpath.2015.02.010

29. Wilson GS, Hu Z, Duan W, Tian A, Wang XM, McLeod D, et al. Efficacy of using cancer stem cell markers in isolating and characterizing liver cancer stem cells. Stem Cells Dev. (2013) 22:2655-64. doi: 10.1089/scd.2012.0703

30. Baguley BC. Multiple drug resistance mechanisms in cancer. Mol Biotechnol. (2010) 46:308-16. doi: 10.1007/s12033-010-9321-2

31. Li X, Lewis MT, Huang J, Gutierrez C, Osborne CK, Wu MF, et al. Intrinsic resistance of tumorigenic breast cancer cells to chemotherapy. J Natl Cancer Inst. (2008) 100:672-9. doi: 10.1093/jnci/djn123

32. Ginestier C, Hur MH, Charafe-Jauffret E, Monville F, Dutcher J, Brown $\mathrm{M}$, et al. ALDH1 is a marker of normal and malignant human mammary stem cells and a predictor of poor clinical outcome. Cell Stem Cell. (2007) 1:555-67. doi: 10.1016/j.stem.2007.08.014

33. Singh SK, Hawkins C, Clarke ID, Squire JA, Bayani J, Hide T, et al Identification of human brain tumour initiating cells. Nature. (2004) 432:396-401. doi: 10.1038/nature03128

34. Gammella E, Recalcati S, Cairo G. Dual Role of ROS as signal and stress agents: iron tips the balance in favor of toxic effects. Oxida Med Cell Longevity. (2016) 2016:1-9. doi: 10.1155/2016/8629024

35. Recalcati S, Gammella E, Buratti P, Cairo G. Molecular regulation of cellular iron balance. IUBMB Life. (2017) 69:389-98. doi: 10.1002/iub.1628

36. Fonseca-Nunes A, Jakszyn P, Agudo A. Iron and cancer risk-a systematic review and meta-analysis of the epidemiological evidence. Cancer Epidemiol Biomarkers Prev. (2014) 23:12-31. doi: 10.1158/1055-9965.EPI-13-0733

37. Pietrangelo A, Rocchi E, Ferrari A, Ventura E, Cairo G. Regulation of hepatic transferrin, transferrin receptor and ferritin genes in human siderosis. Hepatology. (1991) 14:1083-9. doi: 10.1002/hep.1840140623

38. Niederau C, Fischer R, Sonnenberg A, Stremmel W, Trampisch HJ, Strohmeyer G. Survival and causes of death in cirrhotic and in noncirrhotic 
patients with primary hemochromatosis. N Engl J Med. (1985) 313:1256-62. doi: 10.1056/NEJM198511143132004

39. Torti SV, Torti FM. Iron and cancer: more ore to be mined. Nat Rev Cancer. (2013) 13:342-55. doi: 10.1038/nrc3495

40. Manz DH, Blanchette NL, Paul BT, Torti FM, Torti SV. Iron and cancer: recent insights. Ann N Y Acad Sci. (2016) 1368:149-61. doi: $10.1111 /$ nyas. 13008

41. Spangler B, Morgan CW, Fontaine SD, Vander Wal MN, Chang CJ, Wells JA, et al. A reactivity-based probe of the intracellular labile ferrous iron pool. Nat Chem Biol. (2016) 12:680-5. doi: 10.1038/nchembio. 2116

42. Tejedor JR, Papasaikas P, Valcárcel J. Genome-wide identification of Fas/CD95 alternative splicing regulators reveals links with iron homeostasis. Mol Cell. (2015) 57:23-38.

43. Zhang J, Chen X. p53 tumor suppressor and iron homeostasis. FEBS J. (2019) 286:620-9. doi: 10.1111/febs.14638

44. Dongiovanni P, Fracanzani AL, Cairo G, Megazzini CP, Gatti S, Rametta R, et al. Iron-dependent regulation of MDM2 influences p53 activity and hepatic carcinogenesis. Am J Pathol. (2010) 176:1006-17. doi: 10.2353/ajpath.2010.090249

45. Shen J, Sheng X, Chang Z, Wu Q, Wang S, Xuan Z, et al. Iron metabolism regulates $\mathrm{p} 53$ signaling through direct heme-p 53 interaction and modulation of p53 localization, stability, and function. Cell Rep. (2014) 7:180-93. doi: 10.1016/j.celrep.2014.02.042

46. Gammella E, Buratti P, Cairo G, Recalcati S. The transferrin receptor: the cellular iron gate. Metallomics. (2017) 9:1367-75. doi: 10.1039/C7MT00143F

47. Shen Y, Li X, Dong D, Zhang B, Xue Y, Shang P. Transferrin receptor 1 in cancer: a new sight for cancer therapy. Am J Cancer Res. (2018) 8:916-31.

48. Cairo G, Tacchini L, Pietrangelo A. Lack of coordinate control of ferritin and transferrin receptor expression during rat liver regeneration. Hepatology. (1998) 28:173-8. doi: 10.1002/hep.510280123

49. Shen Y, Li X, Zhao B, Xue Y, Wang S, Chen X, et al. Iron metabolism gene expression and prognostic features of hepatocellular carcinoma. J Cell Biochem. (2018) 119:9178-204. doi: 10.1002/jcb.27184

50. Jamnongkan W, Thanan R, Techasen A, Namwat N, Loilome W, Intarawichian $\mathrm{P}$, et al. Upregulation of transferrin receptor-1 induces cholangiocarcinoma progression via induction of labile iron pool. Tumour Biol. (2017) 39:1010428317717655. doi: 10.1177/1010428317717655

51. Raggi C, Gammella E, Correnti M, Buratti P, Forti E, Andersen JB, et al. Dysregulation of iron metabolism in cholangiocarcinoma Stem-like Cells. Sci Rep. (2017) 7:17667. doi: 10.1038/s41598-017-17804-1

52. Wessling-Resnick M. Crossing the iron gate: why and how transferrin receptors mediate viral entry. Annu Rev Nutr. (2018) 38:431-58. doi: 10.1146/annurev-nutr-082117-051749

53. Jian J, Yang Q, Huang X. Src regulates $\operatorname{Tyr}(20)$ phosphorylation of transferrin receptor-1 and potentiates breast cancer cell survival. J Biol Chem. (2011) 286:35708-15. doi: 10.1074/jbc.M111.271585

54. Chen AC, Donovan A, Ned-Sykes R, Andrews NC. Noncanonical role of transferrin receptor 1 is essential for intestinal homeostasis. Proc Natl Acad Sci USA. (2015) 112:11714-9. doi: 10.1073/pnas.1511701112

55. Liang W, Li Q, Ferrara N. Metastatic growth instructed by neutrophilderived transferrin. Proc Natl Acad Sci USA. (2018) 115:11060-5. doi: $10.1073 /$ pnas. 1811717115

56. Udali S, Castagna A, Corbella M, Ruzzenente A, Moruzzi S, Mazzi F, et al. Hepcidin and DNA promoter methylation in hepatocellular carcinoma. Eur J Clin Invest. (2018) 48:e12870. doi: 10.1111/eci.12870

57. Shpyleva SI, Muskhelishvili L, Tryndyak VP, Koturbash I, Tokar EJ, Waalkes MP, et al. Chronic administration of 2-acetylaminofluorene alters the cellular iron metabolism in rat liver. Toxicol Sci. (2011) 123:433-40. doi: $10.1093 /$ toxsci/kfr 193

58. Wang W, Deng Z, Hatcher H, Miller LD, Di X, Tesfay L, et al. IRP2 regulates breast tumor growth. Cancer Res. (2014) 74:497-507. doi: 10.1158/0008-5472.CAN-13-1224

59. Chan JJ, Kwok ZH, Chew XH, Zhang B, Liu C, Soong TW, et al. A FTH1 gene:pseudogene:microRNA network regulates tumorigenesis in prostate cancer. Nucleic Acids Res. (2018) 46:1998-2011. doi: 10.1093/nar/ gkx1248

60. Bomford AB, Munro HN. Ferritin gene expression in health and malignancy. Pathobiology. (1992) 60:10-8. doi: 10.1159/000163691
61. Arosio P, Carmona F, Gozzelino R, Maccarinelli F, Poli M. The importance of eukaryotic ferritins in iron handling and cytoprotection. Biochem J. (2015) 472:1-15. doi: 10.1042/BJ20150787

62. El Hout M, Dos Santos L, Hamaï A, Mehrpour M. A promising new approach to cancer therapy: Targeting iron metabolism in cancer stem cells. Semin Cancer Biol. (2018) 53:125-38. doi: 10.1016/j.semcancer.2018.07.009

63. Wu KJ, Polack A, Dalla-Favera R. Coordinated regulation of ironcontrolling genes, H-ferritin and IRP2, by c-MYC. Science. (1999) 283:676-9. doi: $10.1126 /$ science. 283.5402 .676

64. Schonberg DL, Miller TE, Wu Q, Flavahan WA, Das NK, Hale JS, et al. Preferential Iron trafficking characterizes glioblastoma stem-like cells. Cancer Cell. (2015) 28:441-55. doi: 10.1016/j.ccell.2015.09.002

65. Chitambar CR, Al-Gizawiy MM, Alhajala HS, Pechman KR, Wereley JP, Wujek R, et al. Gallium maltolate disrupts tumor iron metabolism and retards the growth of glioblastoma by inhibiting mitochondrial function and ribonucleotide reductase. Mol Cancer Ther. (2018) 17:1240-50. doi: 10.1158/1535-7163.MCT-17-1009

66. Basuli D, Tesfay L, Deng Z, Paul B, Yamamoto Y, Ning G, et al. Iron addiction: a novel therapeutic target in ovarian cancer. Oncogene. (2017) 36:4089-99. doi: 10.1038/onc.2017.11

67. Kanojia D, Zhou W, Zhang J, Jie C, Lo PK, Wang Q, et al. Proteomic profiling of cancer stem cells derived from primary tumors of HER2/Neu transgenic mice. Proteomics. (2012) 12:3407-15. doi: 10.1002/pmic.201200103

68. Lobello N, Biamonte F, Pisanu ME, Faniello MC, Jakopin Ž, Chiarella E, et al. Ferritin heavy chain is a negative regulator of ovarian cancer stem cell expansion and epithelial to mesenchymal transition. Oncotarget. (2016) 7:62019-33. doi: 10.18632/oncotarget.11495

69. Pourcelot E, Lénon M, Mobilia N, Cahn JY, Arnaud J, Fanchon E, et al. Iron for proliferation of cell lines and hematopoietic progenitors: nailing down the intracellular functional iron concentration. Biochim Biophys Acta. (2015) 1853:1596-605. doi: 10.1016/j.bbamcr.2015.03.009

70. Yamasaki T, Terai S, Sakaida I. Deferoxamine for advanced hepatocellular carcinoma. N Engl J Med. (2011) 365:576-8. doi: 10.1056/NEJMc1 105726

71. Paubelle E, Zylbersztejn F, Alkhaeir S, Suarez F, Callens C, Dussiot M, et al. Deferasirox and vitamin D improves overall survival in elderly patients with acute myeloid leukemia after demethylating agents failure. PLoS ONE. (2013) 8:e65998. doi: 10.1371/journal.pone.0065998

72. Lane DJ, Mills TM, Shafie NH, Merlot AM, Saleh Moussa R, Kalinowski DS, et al. Expanding horizons in iron chelation and the treatment of cancer: role of iron in the regulation of ER stress and the epithelialmesenchymal transition. Biochim Biophys Acta. (2014) 1845:166-81. doi: 10.1016/j.bbcan.2014.01.005

73. Recalcati S, Gammella E, Cairo G. New perspectives on the molecular basis of the interaction between oxygen homeostasis and iron metabolism. Hypoxia. (2015) 3:93-103. doi: 10.2147/HP.S83537

74. Spagnuolo RD, Recalcati S, Tacchini L, Cairo G. Role of hypoxiainducible factors in the dexrazoxane-mediated protection of cardiomyocytes from doxorubicin-induced toxicity. Br J Pharmacol. (2011) 163:299-312. doi: $10.1111 / j .1476-5381.2011 .01208 . x$

75. Moussa RS, Kovacevic Z, Bae DH, Lane DJR, Richardson DR. Transcriptional regulation of the cyclin-dependent kinase inhibitor, p21. Biochim Biophys Acta Gen Subj. (2018) 1862:761-74. doi: 10.1016/j.bbagen.2017.10.009

76. Kalinowski DS, Stefani C, Toyokuni S, Ganz T, Anderson GJ, Subramaniam $\mathrm{NV}$, et al. Redox cycling metals: pedaling their roles in metabolism and their use in the development of novel therapeutics. Biochim Biophys Acta. (2016) 1863:727-48. doi: 10.1016/j.bbamcr.2016.01.026

77. Al-Akra L, Bae DH, Sahni S, Huang MLH, Park KC, Lane DJR, et al. Tumor stressors induce two mechanisms of intracellular P-glycoprotein-mediated resistance that are overcome by lysosomal-targeted thiosemicarbazones. $J$ Biol Chem. (2018) 293:3562-87. doi: 10.1074/jbc.M116.772699

78. Huang T, Sun Y, Li Y, Wang T, Fu Y, Li C, et al. Growth Inhibition of a Novel iron chelator, dpdtc, against hepatoma carcinoma cell lines partly attributed to ferritinophagy-mediated lysosomal ROS generation. Oxid Med Cell Longev. (2018) 2018:4928703. doi: 10.1155/2018/4928703

79. Stockwell BR, Friedmann Angeli JP, Bayir H, Bush AI, Conrad M, Dixon SJ, et al. Ferroptosis: a regulated cell death nexus linking metabolism, redox biology, and disease. Cell. (2017) 171:273-85. doi: 10.1016/j.cell.2017.09.021 
80. Doll S, Conrad M. Iron and ferroptosis: A still ill-defined liaison. IUBMB Life. (2017) 69:423-34. doi: 10.1002/iub.1616

81. Toyokuni S, Ito F, Yamashita K, Okazaki Y, Akatsuka S. Iron and thiol redox signaling in cancer: an exquisite balance to escape ferroptosis. Free Radic Biol Med. (2017) 108:610-26. doi: 10.1016/j.freeradbiomed.2017.04.024

82. Feng H, Stockwell BR. Unsolved mysteries: How does lipid peroxidation cause ferroptosis? PLoS Biol. (2018) 16:e2006203. doi: 10.1371/journal.pbio.2006203

83. Hangauer MJ, Viswanathan VS, Ryan MJ, Bole D, Eaton JK, Matov A, et al. Drug-tolerant persister cancer cells are vulnerable to GPX4 inhibition. Nature. (2017) 551:247-50. doi: 10.1038/nature24297

84. Louandre C, Ezzoukhry Z, Godin C, Barbare JC, Mazière JC, Chauffert B, et al. Iron-dependent cell death of hepatocellular carcinoma cells exposed to sorafenib. Int J Cancer. (2013) 133:1732-42. doi: 10.1002/ijc.28159

85. Sun X, Ou Z, Chen R, Niu X, Chen D, Kang R, et al. Activation of the p62-Keap1-NRF2 pathway protects against ferroptosis in hepatocellular carcinoma cells. Hepatology. (2016) 63:173-84. doi: 10.1002/hep.28251

86. Carnero A, Garcia-Mayea Y, Mir C, Lorente J, Rubio IT, LLeonart ME. The cancer stem-cell signaling network and resistance to therapy. Cancer Treat Rev. (2016) 49:25-36. doi: 10.1016/j.ctrv.2016.07.001

87. Mai TT, Hamaï A, Hienzsch A, Cañeque T, Müller S, Wicinski J, et al. Salinomycin kills cancer stem cells by sequestering iron in lysosomes. Nat Chem. (2017) 9:1025-33. doi: 10.1038/NCHEM.2778

88. Campanella A, Santambrogio P, Fontana F, Frenquelli M, Cenci $S$, Marcatti $M$, et al. Iron increases the susceptibility of multiple myeloma cells to bortezomib. Haematologica. (2013) 98:971-9. doi: 10.3324/haematol.2012.074872

89. Cenci S, Sitia R. Managing and exploiting stress in the antibody factory. FEBS Lett. (2007) 581:3652-7. doi: 10.1016/j.febslet.2007.04.031

90. Lv H, Wang C, Fang T, Li T, Lv G, Han Q, et al. Vitamin C preferentially kills cancer stem cells in hepatocellular carcinoma via SVCT-2. NPJ Precis Oncol. (2018) 2:1. doi: 10.1038/s41698-017-0044-8

91. Andrews NC, Schmidt PJ. Iron homeostasis. Annu Rev Physiol. (2007) 69:69-85. doi: 10.1146/annurev.physiol.69.031905.164337

92. Aslam MF, Frazer DM, Faria N, Bruggraber SF, Wilkins SJ, Mirciov $\mathrm{C}$, et al. Ferroportin mediates the intestinal absorption of iron from a nanoparticulate ferritin core mimetic in mice. FASEB J. (2014) 28:3671-8. doi: 10.1096/fj.14-251520

93. Girelli D, Ugolini S, Busti F, Marchi G, Castagna A. Modern iron replacement therapy: clinical and pathophysiological insights. Int J Hematol. (2018) 107:16-30. doi: 10.1007/s12185-017-2373-3

94. Gustafson HH, Holt-Casper D, Grainger DW, Ghandehari H. Nanoparticle Uptake: The Phagocyte Problem. Nano Today. (2015) 10:487-510. doi: 10.1016/j.nantod.2015.06.006

95. Shi L, Ito F, Wang Y, Okazaki Y, Tanaka H, Mizuno M, et al. Non-thermal plasma induces a stress response in mesothelioma cells resulting in increased endocytosis, lysosome biogenesis and autophagy. Free Radic Biol Med. (2017) 108:904-17. doi: 10.1016/j.freeradbiomed.2017.04.368

96. Yang ND, Tan SH, Ng S, Shi Y, Zhou J, Tan KS, et al. Artesunate induces cell death in human cancer cells via enhancing lysosomal function and lysosomal degradation of ferritin. J Biol Chem. (2014) 289:33425-41. doi: 10.1074/jbc.M114.564567

97. Zhang Z, Yao Z, Wang L, Ding H, Shao J, Chen A, et al. Activation of ferritinophagy is required for the RNA-binding protein ELAVL1/HuR to regulate ferroptosis in hepatic stellate cells. Autophagy. (2018) 14:2083-103. doi: 10.1080/15548627.2018.1503146

98. Maccarinelli F, Gammella E, Asperti M, Regoni M, Biasiotto G, Turco $\mathrm{E}$, et al. Mice lacking mitochondrial ferritin are more sensitive to doxorubicin-mediated cardiotoxicity. J Mol Med. (2014) 92:859-69. doi: 10.1007/s00109-014-1147-0

99. Recalcati S, Locati M, Marini A, Santambrogio P, Zaninotto F, De Pizzol $\mathrm{M}$, et al. Differential regulation of iron homeostasis during human macrophage polarized activation. Eur J Immunol. (2010) 40:824-35. doi: 10.1002/eji.200939889

100. Corna G, Campana L, Pignatti E, Castiglioni A, Tagliafico E, Bosurgi $\mathrm{L}$, et al. Polarization dictates iron handling by inflammatory and alternatively activated macrophages. Haematologica. (2010) 95:1814-22. doi: 10.3324/haematol.2010.023879

101. Cairo G, Recalcati S, Mantovani A, Locati M. Iron trafficking and metabolism in macrophages: contribution to the polarized phenotype. Trends Immunol. (2011) 32:241-7. doi: 10.1016/j.it.2011.03.007

102. Thorsson V, Gibbs DL, Brown SD, Wolf D, Bortone DS, Ou Yang TH, et al. The immune landscape of cancer. Immunity. (2018) 48:812-30.e814. doi: 10.1016/j.immuni.2018.03.023

103. Leftin A, Zhao H, Turkekul M, de Stanchina E, Manova K, Koutcher JA. Iron deposition is associated with differential macrophage infiltration and therapeutic response to iron chelation in prostate cancer. Sci Rep. (2017) 7:11632. doi: 10.1038/s41598-017-11899-2

104. Leftin A, Ben-Chetrit N, Klemm F, Joyce JA, Koutcher JA. Iron imaging reveals tumor and metastasis macrophage hemosiderin deposits in breast cancer. PLoS ONE. (2017) 12:e0184765. doi: 10.1371/journal.pone.0 184765

105. Madeddu C, Gramignano G, Kotsonis P, Coghe F, Atzeni V, Scartozzi $\mathrm{M}$, et al. Microenvironmental M1 tumor-associated macrophage polarization influences cancer-related anemia in advanced ovarian cancer: key role of interleukin-6. Haematologica. (2018) 103:e388-91. doi: 10.3324/haematol.2018.191551

106. Costa da Silva M, Breckwoldt MO, Vinchi F, Correia MP, Stojanovic A, Thielmann CM, et al. Iron induces anti-tumor activity in tumor-associated macrophages. Front Immunol. (2017) 8:1479. doi: 10.3389/fimmu.2017.01479

107. Biswas SK, Allavena P, Mantovani A. Tumor-associated macrophages: functional diversity, clinical significance, and open questions. Semin Immunopathol. (2013) 35:585-600. doi: 10.1007/s00281-0130367-7

108. Miller LD, Coffman LG, Chou JW, Black MA, Bergh J, D’Agostino R, et al. An iron regulatory gene signature predicts outcome in breast cancer. Cancer Res. (2011) 71:6728-37. doi: 10.1158/0008-5472.CAN-11-1870

109. Zhang S, Chen Y, Guo W, Yuan L, Zhang D, Xu Y, et al. Disordered hepcidinferroportin signaling promotes breast cancer growth. Cell Signal. (2014) 26:2539-50. doi: 10.1016/j.cellsig.2014.07.029

110. Pfeifhofer-Obermair C, Tymoszuk P, Petzer V, Weiss G, Nairz M. Iron in the Tumor Microenvironment-Connecting the Dots. Front Oncol. 8:549. doi: $10.3389 /$ fonc. 2018.00549

111. Laskar A, Eilertsen J, Li W, Yuan XM. SPION primes THP1 derived M2 macrophages towards M1-like macrophages. Biochem Biophys Res Commun. (2013) 441:737-42. doi: 10.1016/j.bbrc.2013.1 0.115

112. Zanganeh S, Hutter G, Spitler R, Lenkov O, Mahmoudi M, Shaw A, et al. Iron oxide nanoparticles inhibit tumour growth by inducing pro-inflammatory macrophage polarization in tumour tissues. Nat Nanotechnol. (2016) 11:98694. doi: $10.1038 /$ nnano.2016.168

Conflict of Interest Statement: The authors declare that the research was conducted in the absence of any commercial or financial relationships that could be construed as a potential conflict of interest.

Copyright (C) 2019 Recalcati, Correnti, Gammella, Raggi, Invernizzi and Cairo. This is an open-access article distributed under the terms of the Creative Commons Attribution License (CC BY). The use, distribution or reproduction in other forums is permitted, provided the original author(s) and the copyright owner(s) are credited and that the original publication in this journal is cited, in accordance with accepted academic practice. No use, distribution or reproduction is permitted which does not comply with these terms. 